\title{
Colorectal Neuroendocrine Tumor G1
}

National Cancer Institute

\section{Source}

National Cancer Institute. Colorectal Neuroendocrine Tumor G1. NCI Thesaurus. Code C96160.

A well differentiated, low grade neuroendocrine tumor (carcinoid tumor) that arises from the colon or rectum. The mitotic count is less than 2 per 10 HPF and/or the Ki67 index is equal to or less than 2 percent. 